CERN-EP/84-159

26 November 1984

\title{
THE $\left(\mathrm{K}^{-}, \pi^{+}\right)$STRANGENESS EXCHANGE REACTION ON ${ }^{16} \mathrm{O}$
}

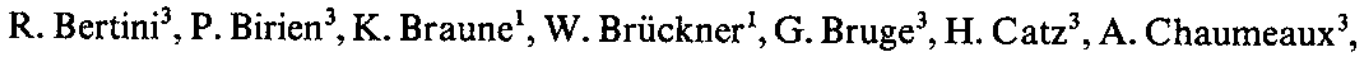 \\ J. Ciborowski ${ }^{2}$, H. Döbbeling ${ }^{1}$, J.M. Durand ${ }^{3}$, R.W. Frey ${ }^{2}$, D. Garreta ${ }^{3}$, S. Janouin ${ }^{3}$, \\ T.J. Ketel ${ }^{1}$, K. Kilian ${ }^{1}$,'H. Kneis ${ }^{1}$, S. Majewski ${ }^{2}$, B. Mayer ${ }^{3}$, J.C. Peng ${ }^{3,4}$, \\ B. Povh ${ }^{1}$, R.D. Ransome ${ }^{1}$, R. Szwed ${ }^{1}$, T.-A. Shibata ${ }^{2}$, A. Thiessen ${ }^{1,4}$, \\ M. Treichel ${ }^{1}$, M. Uhrmacher ${ }^{1}$ and Th. Walcher ${ }^{1}$ \\ Heidelberg*-Saclay Collaboration
}

\begin{abstract}
Using negative kaons of $450 \mathrm{MeV} / \mathrm{c}$ momentum, $\Sigma$ hypernuclear states have been produced in the $\left(\mathrm{K}^{-}, \pi^{+}\right)$reaction on ${ }^{16} \mathrm{O}$. The observed states are interpreted as the recoilless production of $\Sigma^{-}$ particles in the $p_{3 / 2}$ and $p_{1 / 2}$ orbits of the ${ }_{\Sigma}^{16} C$ hypernucleus. Their energy splitting gives a constraint to the magnitude of the spin-orbit coupling for the $\Sigma^{-}$particles in the nucleus.
\end{abstract}

(Submitted to Physics Letters B)

${ }^{1}$ Max-Planck-Institut für Kernphysik, Heidelberg, Fed. Rep. Germany.

2 Physikalisches Institut der Universität, Heidelberg, Fed. Rep. Germany.

${ }^{3} \mathrm{DPhN} / \mathrm{ME}, \mathrm{CEN}$, Saclay, France.

4 On leave from Los Alamos National Laboratory, NM, USA.

* Supported by the Bundesministerium für Forschung und Technologie, Germany. 


\section{INTRODUCTION}

In a recent paper [1] we presented the results on $\Sigma$ hypernuclear states produced in the $\left(\mathrm{K}^{-}\right.$, $\pi^{+}$) reaction on a ${ }^{12} \mathrm{C}$ target. Only substitutional states which could be most naturally interpreted as $\Sigma^{+}, \Sigma^{0}$, and $\Sigma^{-}$replacing a proton or a neutron in the $p_{3 / 2}$ shell have been observed. A surprisingly small width of these states of less than $5 \mathrm{MeV}$ has been determined from these measurements. The width of the $\Sigma$ hypernuclear states is believed to be determined by the $\Sigma+N \rightarrow \Lambda+N$ conversion in nuclei, which should produce a width of about $25 \mathrm{MeV}$ [2]. Different quenching mechanisms [2,3] of this reaction have to be involved if the lárge $\Sigma$ lifetime in nuclei is a general phenomenon. The narrowness may be just limited to the states observed so far, which are without exception slightly above the $\Sigma$-particle threshold. None of the $\Sigma$ hypernuclear ground states $\left(\mathrm{s}_{1 / 2}, \mathrm{p}_{3 / 2}^{-1}\right)_{\Sigma, \mathrm{N}}$ has been identified. It is not clear whether the non-observation of the ground states is due to their large width or whether it is just the consequence of the relatively poor statistics which could be collected in the first experiments.

Even though we do not understand the smallness of the width of the observed $\Sigma$ hypernuclear states, some general features of the $\Sigma$-nucleus interaction can be deduced from these data. In particular the potential depth for $\Sigma$ particles in the nucleus is about $20 \mathrm{MeV}$ [4] as compared to the $\Lambda$ one of about $28 \mathrm{MeV}$ and the nucleon one of about $50 \mathrm{MeV}$.

In the present paper we report on the $\left(\mathrm{K}^{-}, \pi^{+}\right)$reaction on the ${ }^{16} \mathrm{O}$ target, which has been performed with the aim of estimating the spin-orbit interaction for the $\Sigma$ particles in the nucleus. In the case of the $\Lambda$ hypernuclei the comparison between the ${ }_{\Lambda}^{12} \mathrm{C}$ and ${ }_{\Lambda}^{16} \mathrm{O}$ spectra has been used very successfully to deduce the spin-orbit interaction for the $\Lambda$ particle in the nucleus [5].

\section{EXPERIMENTAL SET-UP AND ANALYSIS}

The experiment has been performed at the low-momentum kaon beam $k_{26}$ of the CERN Proton Synchrotron (PS). The $\mathrm{k}_{26}$ beam is drastically shorter (the length being only $11.5 \mathrm{~m}$ ) than the $\mathrm{k}_{24}$ beam used in the $\Lambda$ hypernuclear experiments [6] since the recoilless production of $\Sigma$ hypernuclei requires a lower $\mathrm{K}$ momentum ( $\leq 450 \mathrm{MeV} / \mathrm{c}$ ). A sufficient separation of $\mathrm{K}$ 's from $\pi$ 's was achieved by second-order corrections of the imaging of the separated beam. In this way an angular acceptance of $7 \mathrm{msr}$ and a momentum bite of $\pm 2.5 \%$ were transmissible. The detector arrangement was essentially the same as the one used in the $\Lambda$ hypernuclear experiments.

The present experiment has been performed with negative kaons of $450 \mathrm{MeV} / \mathrm{c}$ momentum. At this momentum and with the $3 \times 10^{12}$ protons on the production target, $10^{4}$ negative kaons and $2 \times 10^{5}$ pions per machine pulse ( $300 \mathrm{~ms}$ length) passed the beam separation stage and reached the experimental target. The reaction trigger has to guarantee that the particle was a kaon before the target and a pion after the target. Therefore kaons were further selected after the separation stage by their time of flight in the beam spectrometer and their $\mathrm{dE}$ in the time-of-flight scintillation counters. A liquid-hydrogen Cherenkov counter in front of the target was used to suppress the pion contamination of the $\mathrm{K}$ decays in the beam.

The reaction trigger was rather clean, as the only source of the positive pions in the energy region expected for the transitions to the $\Sigma$ hypernuclear states stems from the $\left(\mathrm{K}^{-}, \pi^{+}\right)$strangeness exchange reaction. The $\left(\mathrm{K}^{-}, \pi^{+}\right)$reaction on hydrogen of the liquid-hydrogen Cherenkov counter gives a strong signal corresponding to the free $\Sigma^{-}$production. In the presented spectra it was suppressed in the off-line analysis. Owing to the large momentum byte of the pion spectrometer, also 
positive pions from the $\mathrm{K}^{-} \rightarrow 3 \pi$ decay have been observed but they are kinematically well separated from the $\Sigma$ hypernuclear lines.

As oxygen target a $2 \mathrm{~cm}$ thick cryotarget was mounted in the same vacuum system as the liquid-hydrogen Cherenkov counter. Their walls were separated by $4 \mathrm{~cm}$.

In the off-line analysis, we carry out a complete vertex reconstruction at the target and at the exit of the pion spectrometer. With the help of this reconstruction it is possible to reject almost all events coming not from the target. Owing to the high rate for the $\mathrm{K}^{-}+\mathrm{p} \rightarrow \pi^{+}+\Sigma^{-}$reaction on the liquid-hydrogen Cherenkov counter (this rate is about ten times higher than the reaction rate on the ${ }^{16} \mathrm{O}$ target) the suppression is not complete. In the spectra the remainder of the $\Sigma^{-}$line is indicated and gives an impression of the efficiency of the vertex reconstruction. Imposing a geometrical window on the liquid-hydrogen Cherenkov counter, one selects the $\mathrm{K}^{-}+\mathrm{p} \rightarrow \pi^{+}+\Sigma^{-}$reaction. This allows the energy calibration to be verified and the resolution of the system to be determined. The mass resolution is $3 \mathrm{MeV}$ FWHM and is predominantly determined by the target thickness.

\section{3. $\quad \Sigma^{-}$SUBSTITUTIONAL STATES IN ${ }_{\Sigma}^{16} \mathrm{C}$}

Figure $1 \mathrm{~b}$ shows a spectrum from the $\left(\mathrm{K}^{-}, \pi^{+}\right)$reaction on ${ }^{16} \mathrm{O}$ leading to $\Sigma^{-}$production. This spectrum is to be compared to the one obtained on ${ }^{12} \mathrm{C}[1]$ and is shown in fig. 1a. The peak in the ${ }_{2}^{12} \mathrm{Be}$ spectrum observed at $279 \pm 1 \mathrm{MeV}$ is ascribed to the substitutional state with a configuration $\left(\mathrm{p}_{3 / 2}, \mathrm{p}_{3 / 2}^{-1 / 2}\right)_{\Sigma^{-}, \mathrm{p}}$. In the ${ }_{\Sigma}^{16} \mathrm{C}$ spectrum two transitions are observed, one at $277.5 \pm 1 \mathrm{MeV}$ and the other at $284 \pm 1 \mathrm{MeV}$. They presumably belong to the $\left(\mathrm{p}_{3 / 2, \mathrm{p}_{3 / 2}}^{-1}\right)_{\Sigma^{-}, \mathrm{p}}$ and $\left(\mathrm{p}_{1 / 2}, \mathrm{p}_{1 / 2}^{-1}\right)_{\Sigma^{-}, \mathrm{p}}$ configurations. In order to deduce their relative intensities, the line shape of the structure in ${ }_{2}^{12} \mathrm{Be}$ was assumed to be composed of a narrow line at $279 \mathrm{MeV}$ due to the $\left(\mathrm{p}_{3 / 2}, \mathrm{p}_{3 / 2}^{-1}\right)_{\Sigma^{-}, \mathrm{p}}$ configuration and a broad shoulder around $285 \mathrm{MeV}$ due to quasi-free transitions, i.e. transitions to higher shells. The curve through the lower mass peak in ${ }_{\Sigma}^{16} \mathrm{C}$ shows an estimate of this shape. In particular, the quasi-free contribution has to be regarded as approximative only. On top of this a normal resonance shape at $284 \mathrm{MeV}$ reproduces the measured spectrum. From this fit an intensity ratio of $1.5 \pm 0.3$ for the lower peak to the upper peak is estimated.

A comparison between the mass spectra of neighbouring $\Sigma$ hypernuclei is particularly instructive if they are plotted against the mass scale $M_{H Y}-M_{A}$, i.e. the difference of the hypernuclear mass minus the mass of the target nucleus. For moderate residual $\Sigma \mathrm{N}$ interaction, i.e. smaller than the shell spacing for the $\Sigma$ particle and smaller than the nuclear spin-orbit splitting, the transitions to the states in neighbouring nuclei with the same configuration appear at the same $\mathrm{M}_{\mathrm{HY}}-\mathrm{M}_{\mathrm{A}}$.

In order to compare the transitions to the ${ }_{\Sigma}^{12} \mathrm{Be}$ and ${ }_{\Sigma}^{16} \mathrm{C}$, we have to correct the mass values for the trivial Coulomb energy shift. By replacing a proton by the negative $\Sigma$ in ${ }_{\Sigma}^{16} \mathrm{C}$, the $\Sigma^{-}$will be more bound by $1.5 \mathrm{MeV}$ as compared to the ${ }_{\Sigma}^{12} \mathrm{Be}$. Taking this shift into account, the $279 \mathrm{MeV}$ state of ${ }_{\Sigma}^{12} \mathrm{Be}$ coincides with the $277.5 \mathrm{MeV}$ state of the ${ }_{\Sigma}^{16} \mathrm{C}$ within the experimental errors. Within the assumptions mentioned above, it can be concluded that the $277.5 \mathrm{MeV}$ peak corresponds to the $\left(\mathrm{p}_{3 / 2}, \mathrm{p}_{3 / 2}^{-1}\right)_{\Sigma^{-}, \mathrm{p}}$ and the peak at $284 \mathrm{MeV}$ to the $\left(\mathrm{p}_{1 / 2}, \mathrm{p}_{1 / 2}^{-1}\right)_{\mathrm{\Sigma}^{-}, \mathrm{p}}$ configuration. The intensity ratio of $1.5: 1$ instead of the value derived from the statistical weights of $2: 1$ may be explained by a mixing of the two configurations by residual interaction.

It is important to note that the $\left(\mathrm{p}_{1 / 2}, \mathrm{p}_{1 / 2}^{-1}\right)_{\mathbf{E}^{-}, \mathrm{p}}$ state appears at a mass which is about $6 \mathrm{MeV}$ higher than that for the $\left(\mathrm{p}_{3 / 2}, \mathrm{p}_{3 / 2}^{-1}\right)_{\Sigma^{-}, \mathrm{p}}$ state. This is just the reverse of the siuation in $\Lambda$ hypernuclei.

The spin-orbit interaction for the $\Lambda$ particle in the nucleus is much smaller than the nucleon one [5]. Therefore the splitting between the $\mathrm{p}_{3 / 2}$ and $\mathrm{p}_{1 / 2}$ substitutional state is determined by the 
spin-orbit splitting for the p-shell nucleon in the ${ }^{16} \mathrm{O}$ nucleus. As the $\mathrm{p}_{3 / 2}$ state is bound more strongly than the $\mathrm{p}_{1 / 2}$, the $\left(\mathrm{p}_{1 / 2}, \mathrm{p}_{1 / 2}^{-1}\right)_{\Lambda, \mathrm{n}}$ configuration appears at a lower mass than the $\left(\mathrm{p}_{3 / 2}, \mathrm{p}_{3 / 2}^{-1}\right)_{\Lambda, \mathrm{n}}$ one. Following the same argument for the $\Sigma$ hypernuclei, one would conclude that the spin-orbit interaction for the $\Sigma$ particle is so large as to reverse the order of the $\left(\mathrm{p}_{3 / 2}, \mathrm{p}_{3 / 2}^{-1}\right)_{\Sigma^{-}, \mathrm{p}}$ and the $\left(\mathrm{p}_{1 / 2}, \mathrm{p}_{1 / 2}^{-1}\right)_{\Sigma^{-}, \mathrm{p}}$ states and is thus larger than that of the nucleon one. In fact, by neglecting the residual interaction fully, the spin-orbit interaction for the $\Sigma$ particle would be just $12 \mathrm{MeV}$ or twice that for the nucleon.

The above considerations lose their validity if the expectation value for the residual interaction matrix element is larger than the nuclear spin-orbit splitting. In this case also for a zero spin-orbit interaction for the $\Sigma$ particle in the nucleus one could fit the observed energy spectra. It should be pointed out, however, that in the $\mathrm{p}$ shell the nuclear matrix elements for the residual interaction have a typical value of $2 \mathrm{MeV}$ [7]. It seems to be very unlikely that the residual interaction for the $\Sigma \mathrm{N}$ system would be stronger than that for the $\mathrm{NN}$ system. The $\Sigma \mathrm{N}$ interaction [8] is, in all spin and isospin states, much weaker than the NN one, with the exception of the spin 1 and isospin $1 / 2$ state for which it is comparable in strength to the NN interaction.

Therefore, it seems reasonable to conclude that the results on the $p_{3 / 2}$ and the $p_{1 / 2}$ orbit splitting in ${ }_{\Sigma}^{16} \mathrm{C}$ presented here favour the solutions for the spin-orbit interaction for the $\Sigma$ particle being stronger than that for the nucleon. One should, however, be aware of the fact that this result is obtained by the assumption that the residual interaction is not anomalously strong, i.e. not stronger than the nuclear spin-orbit splitting in the p-shell nuclei. 


\section{REFERENCES}

[1] R. Bertini, P. Birien, K. Braune, W. Brückner, G. Bruge, H. Catz, A. Chaumeaux, J. Ciborowski, H. Döbbeling, J.M. Durand, R.W. Frey, D. Garreta, S. Janouin, T.J. Ketel, K. Kilian, H. Kneis, S. Majewski, B. Mayer, J.C. Peng, B. Povh, R.D. Ransome, R. Szwed, T.-A. Shibata, A. Thiessen, M. Treichel, M. Uhrmacher and Th. Walcher, Phys. Lett. 136B (1984) 29.

[2] A. Gal and C.B. Dover, Phys. Rev. Lett. 44 (1980) 379 and 962.

[3] L.S. Kisslinger, Phys. Rev. Lett. 44 (1980) 968.

L.N. Bogdanova and V.E. Markuskin, Sov. Phys. JETP Lett. 32 (1980) 330.

W. Stepien-Rudzka and S. Wycech, Nucl. Phys. A362(1981) 349.

J. Dabrowski and F. Rozynek, Phys. Rev. C23 (1981) 1706.

A. Gal, G. Toker and U. Alexander, Ann. Phys. 137(1981) 341.

J.A. Johnston and A.W. Thomas, Nucl. Phys. A392(1983) 409.

[4] A. Bouyssy, in Proc. Int. Conf. on Hypernuclear and Kaon Physics, Heidelberg, 1982 (ed.

B. Povh) (MPI Heidelberg Report No. MPI 4-1982-V20), p. 11.

[5] W. Brückner, M.A. Faessler, T.J. Ketel, K. Kilian, J. Niewisch, B. Pietrzyk, B. Povh, H.G. Ritter, M. Uhrmacher, P. Birien, H. Catz, A. Chaumeaux, J.M. Durand, B. Mayer, J. Thirion, R. Bertini and O. Bing, Phys. Lett. 73B (1978) 157.

[6] R. Bertini, O. Bing, P. Birien, K. Braune, W. Brückner, H. Catz, A. Chaumeaux, M.A. Faessler, R.-W. Frey, D. Garreta, T.J. Ketel, K. Kilian, B. Mayer, J. Niewisch, B. Pietrzyk, B. Povh, H.G. Ritter and M. Uhrmacher, Nucl. Phys. A360 (1981) 315.

[7] See, for example, A. Bohr and B. Mottelson, Nuclear Structure (W.A. Benjamin, Inc., New York and Amsterdam, 1979), Vol. I, Paragraph 2.5.

T. Terasawa, Progr. Theor. Phys. 23 (1960) 87.

[8] H.G. Dosch, V.F. Filthuth, V. Hepp and E. Kluge, Phys. Lett. 21 (1966) 236. 


\section{Figure caption}

Fig. $1 . \Sigma$ hypernuclear spectra from ${ }^{12} \mathrm{C}$ (a) and ${ }^{16} \mathrm{O}$ (b) measured at $450 \mathrm{MeV} / \mathrm{c} \mathrm{K}$ momentum. The spectra are plotted as a function of the difference of the hypernuclear mass $\mathrm{m}_{\mathrm{HY}}$ and the target mass $m_{A}$ in $\mathrm{MeV} / \mathrm{c}^{2}$. The continuum on the right-hand side of the dashed line is due to the $\mathrm{K}^{-} \rightarrow 3 \pi$ decay. At $261 \mathrm{MeV} / \mathrm{c}^{2}$ the residue of the $\mathrm{H}\left(\mathrm{K}^{-}, \pi^{+}\right) \Sigma^{-}$reaction from the $\mathrm{LH}_{2}$ Cherenkov detector is visible. The solid lines indicate the line shapes used for fitting the spectra. 


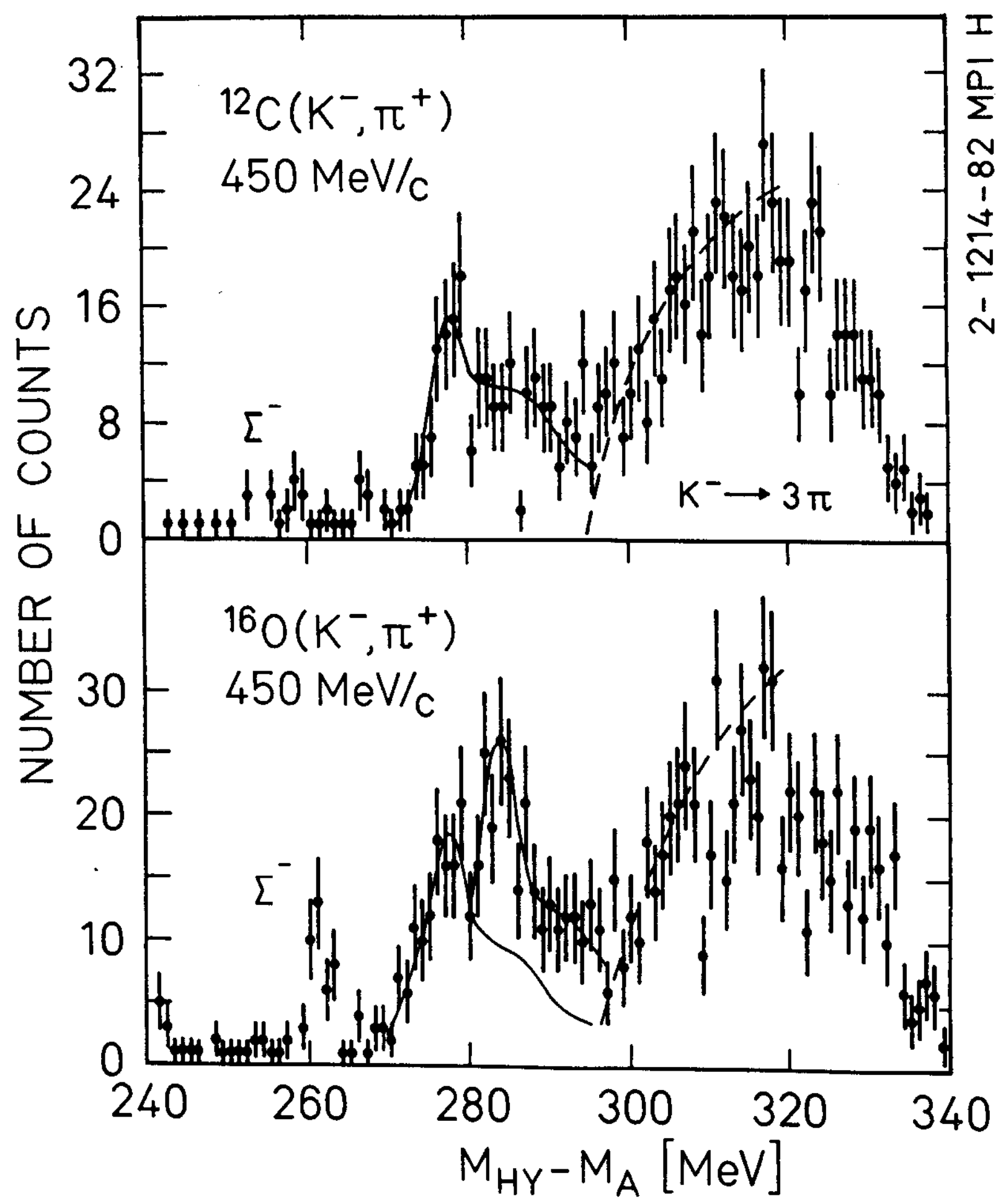

Fig. 1 